\title{
Autoimmune polyglandular syndrome and pulmonary arterial hypertension
}

\section{To the Editors:}

Pulmonary arterial hypertension (PAH) is a serious disease. New treatments have favourably changed the prognosis. Its frequent association with autoimmune thyroid disease (ATD) has been previously acknowledged [1-3]. ATD may also be associated with other autoimmune diseases. Here, we report two cases of PAH associated with type II autoimmune polyglandular syndrome (APS).

In the first case, a 50-yr-old female was diagnosed with idiopathic PAH (dyspnoea on exertion, angina, and mean pulmonary arterial pressure (mPAP) determined by right catheterisation $6.38 \mathrm{kPa}(48 \mathrm{mmHg}))$. Slightly high levels of thyrotropin (TSH) were detected, with normal free thyroxine (FT4) levels, and high serum concentrations of anti-thyroglobulin and anti-thyroid peroxidase antibodies. She improved with i.v. epoprostenol, although it was later necessary to add sildenafil and bosentan. In October 2003, a goitre appeared. Levels of TSH and FT4 were normal. Scintigraphy showed a diffuse goitre without changes in the tracer uptake. Data were concordant with chronic thyroiditis. In August 2004, she presented with polyuria and polydipsia and was admitted to Virgen del Rocío University Hospital (Seville, Spain) with severe hyperglycaemia. She was diagnosed with diabetic ketoacidosis and progressed favourably after treatment with insulin. Pancreatic islet-cell antibodies (ICA) were positive. The scope of the immunological and endocrinological studies was broadened, with the following results: positive anti-adrenal, anti-ovarian, anti-gliadin, antiendomysial and anti-intrinsic factor antibodies, without adrenal insufficiency, ovarian disfunction, malabsorption or vitamin B12 deficiency.

In the second case, a 31-yr-old female, with a history of smoking and previous treatment with oral contraceptives, presented with asthenia, cold intolerance, dyspnoea on exertion and oedemas. She was diagnosed with hypothyroidism as a result of ATD and improved after treatment with levothyroxine. Nevertheless, the oedemas remained, dyspnoea progressed and the patient became icteric due to stasis liver. Echocardiography revealed $\mathrm{PAH}$, confirmed by right catheterisation (mPAP $9.70 \mathrm{kPa}(73 \mathrm{mmHg}))$. Secondary PAH causes were ruled out. Pernicious anaemia was diagnosed with macrocytic anaemia, low vitamin B12 and normal folic acid levels, positive anti-gastric parietal cell antibodies, characteristic changes in the Schilling test, and normal results for malabsorption study with negative anti-gliadin and antiendomysial antibodies. Treatment with i.v. epoprostenol, anticoagulants and vitamin B12 was initiated. Subsequently, the patient was also given sidenafil followed by bosentan, due to therapeutic failure. Epoprostenol was substituted by subcutaneous treprostinil because of repeated losses of vascular access. Due to the association between ATD and pernicious anaemia, the scope of the immunological and endocrinological studies was broadened with the following results: positive anti-adrenal, ICA and anti-ovarian antibodies, without adrenal insufficiency, diabetes or ovarian dysfunction.

APS is defined by the association of several autoimmune diseases, which are not always endocrinological in nature. There are several clinical forms, all with a genetic basis. Type I APS normally begins in childhood and is characterised by chronic mucotaneous candidiasis, hypoparathyroidism and adrenal insufficiency. It is an autosomal recessive inherited disorder, caused by mutations in the autoimmune regulator gene. These mutations determine the risk for the development of multiple autoimmune disorders (type I diabetes, ATD alopecia, vitiligo, pernicious anaemia, ovarian atrophy, etc.). Type II APS is classically associated with adrenal insufficiency, ATD and type I diabetes, although its range of clinical manifestations varies substantially. Some authors distinguish a type III APS, which would link ATD to other autoimmune organ-specific diseases (but not adrenal insufficiency or diabetes), such as pernicious anaemia, while others believe it to be a mere variant of type II APS [4]. The association between PAH and APS has been previously described. KORNISZEWSKI et al. [5] and BHANSALI et al. [6] found type I APS in both cases, and BARROU et al. [7] type II APS, in a patient with early ovarian failure, renal insufficiency, ATD and positive anti-gastric parietal cell antibodies without pernicious anaemia. Using a PubMed database search, using the descriptors "pulmonary hypertension" and "autoimmune polyglandular syndrome" between 1980 and 2005, we have not found any more described cases of PAH associated with type II APS. All of these authors suggest the participation of autoimmune mechanisms in the development of PAH. This is also supported by the frequency of PAH in patients with connective tissue diseases.

The cases which we have described provide more arguments regarding a possible link between autoimmune polyglandular syndrome and pulmonary arterial hypertension. We are unaware if a possible pathogenic link could exist between them, such as a common genetic basis or shared autoimmune mechanisms.

F.J. García-Hernández, C. Ocaña-Medina, R. González-León, R. Garrido-Rasco and J. Sánchez-Román

Virgen del Rocío University Hospitals, Seville, Spain.

\section{REFERENCES}

1 Chu JW, Kao PN, Faul JL, Doyle RL. High prevalence of autoimmune thyroid disease in pulmonary arterial hypertension. Chest 2002; 122: 1668-1673.

2 Curnock AL, Dweik RA, Higgins BH, Saadi HF, Arroliga AC. High prevalence of hypothyroidism in patients with primary pulmonary hypertension. Am J Med Sci 1999; 318: 289-292. 
3 García Hernández FJ, Ocaña Medina C, Mateos Romero L, et al. Combined treatment with intravenous prostacyclin and sildenafil in patients with pulmonary hypertension: report of 4 cases. Med Clin (Barc) 2004; 122: 64-66.

4 Eisenbarth GS, Gorrlieb PA. Medical progress: autoimmune polyendocrine syndromes. $N$ Engl J Med 2004; 350: 2068-2079.

5 Korniszewski L, Kurzyna M, Stolarski B, Torbicki A, Smerdel A, Ploski R. Fatal primary pulmonary hypertension in a 30-yr-old female with APECED syndrome. Eur Respir J 2003; 22: 709-711.
6 Bhansali A, Kotwal N, Suresh V, Murlidharan R, Chattopadhyay A, Mathur K. Polyglandular autoimmune syndrome type 1 without chronic mucocutaneous candidiasis in a 16 year-old male. J Pediatr Endocrinol Metab 2003; 16: 103-105.

7 Barrou Z, Pehuet-Figoni M, Weber S, Lockhart A, Luton JP. Polyendocrinopathy combined with primary pulmonary arterial hypertension. Presse Med 1989; 18: 963-965.

DOI: 10.1183/09031936.06.00133505

\section{ERRATUM}

“ENDOSCOPIC ULTRASOUND-GUIDED BIOPSY IN THE CHEST". J. JANSSEN, S. GASPARINI AND A. MEHTA. EUR RESPIR J 2006; 27: 238-239.

Unfortunately, the affiliation of S. Gasparini was presented incorrectly, and should have appeared as follows: Azienda Ospedali Riuniti, Ancona, Italy.

DOI: $10.1183 / 09031936.06 .50069605$

\section{AUTHOR CORRECTION}

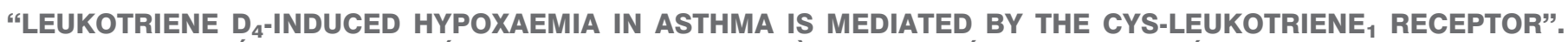
A. CASAS, F.P. GÓMEZ, B. DAHLÉN, J. ROCA, J.A. BARBERÀ, S-E. DAHLÉN AND R. RODRÍGUEZ-ROISIN. EUR RESPIR J 2005; 26: 442-448.

Unfortunately, the above paper has cited an incorrect reference relating to the effects of nifedipine and not $\beta_{2}$-agonists in asthma patients, as stated in the Discussion. Reference 26 should be as follows.

Ballester E, Reyes A, Roca J, Guitart R, Wagner PD, Rodriguez-Roisin R. Ventilation-perfusion mismatching in acute severe asthma: effects of salbutamol and 100\% oxygen. Thorax 1989; 44: 258-267.

The authors apologise sincerelv for this error. 